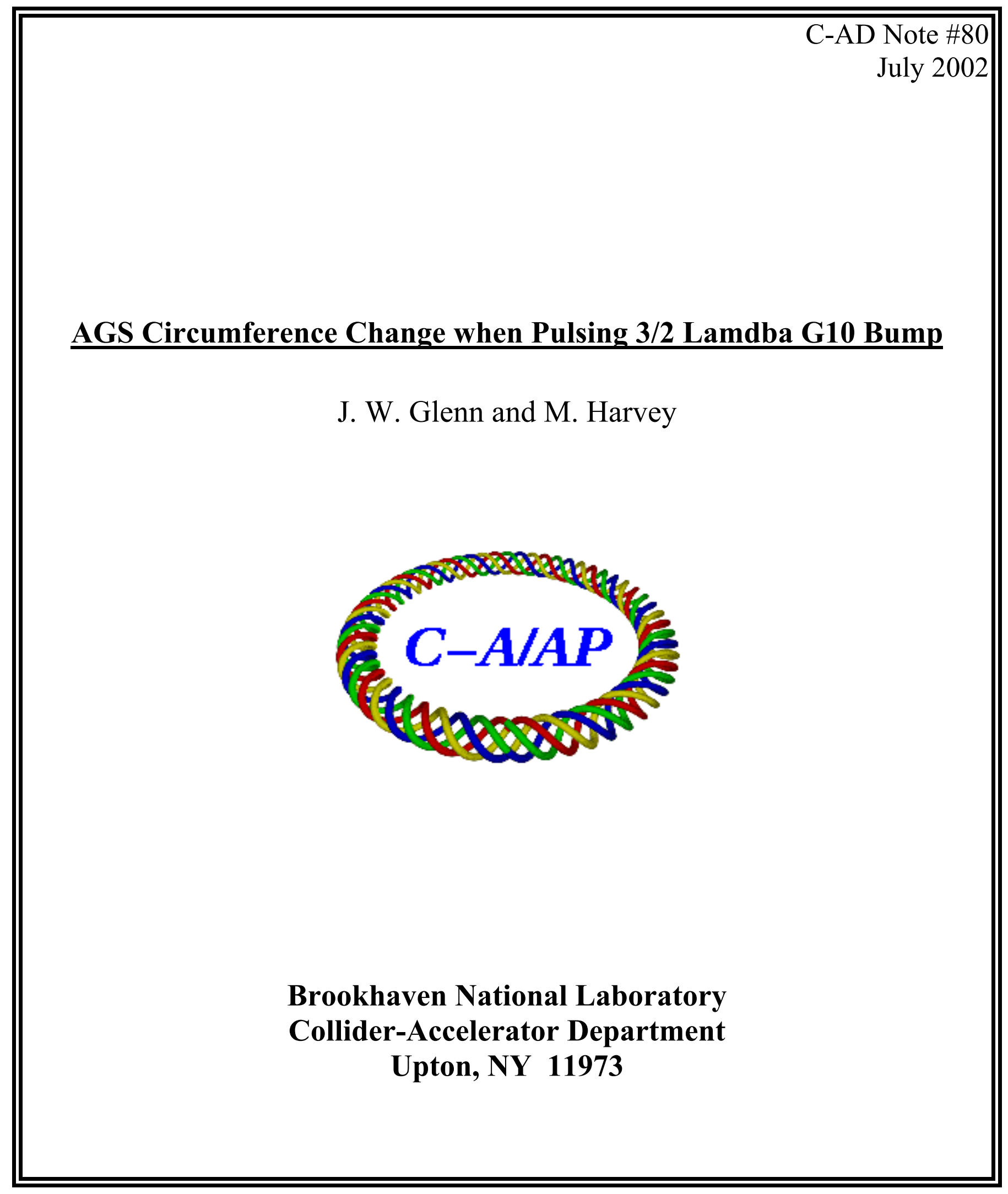


C-A AP Note 80 31July02

J.W. Glenn and M. Harvey

\section{AGS Circumference Change when Pulsing 3/2 Lambda G10 Bump}

\section{Introduction}

The original FEB bumps that move the beam into the G10 kicker and alongside the H10 ejector had two adverse side effects: They lengthen the circumference of the AGS thus requiring deceleration of the beam to keep the frequency constant for RHIC cogging; and they couple to the Main Magnet power supply causing current drift in the magnet. Changing to slowly pulsed 'DC' supplies eliminates these problems but raises concerns at G10 that high intensity power from the beam will be coupled into modulator electronics causing premature failures. All of the circulating beam in the AGS would travel through the G10 kicker magnet for of order hundreds of ms rather than for the few ms of the pulsed configuration. Following from this, the impedance that the high brightness $\mathrm{Au}$ or proton beams see while in the kicker could cause instabilities and dilution. Therefore, a third "hybrid" solution was found that employs a DC bump for the motion at $\mathrm{H} 10$ but retains a modified pulsed bump for G10. In this case the G10 bump is configured to interact only very weakly with the AGS main magnet power supply. Existing windings and power supplies were reconfigured to approximate a $3 / 2$ lambda bump [two magnets were $\sim 25^{\circ}$ out of phase (meaning away from the spacing required for an ideal bump) ].

During the FY2002 HEP we ran tests of the 3/2 lambda G10 Bump at RHIC extraction energy. The windings were powered by existing supplies. We then measured the change in the AGS circumference and AGS field. The bump was configured using the F08, F09, G02, G03 magnets powered by BLWG09A ps and G16, G17, H08, H09 magnets by BLWH09A ps.

\section{Results}

The G10 bump reaches its' maximum amplitude in $\sim 3.5 \mathrm{~ms}$, figure 1 . The bump displaces the horizontal beam orbit by $\sim 20 \mathrm{~mm}$ from the ideal orbit to the inside of the AGS ring at F18, 45 mm to the outside at G08 and $\sim 20 \mathrm{~mm}$ to the inside again at H02. We also see a displacement vertically of $\sim 2 \mathrm{~mm}$ near G08 indicating there may be some coupling. A reference orbit was measured just before turning on the bump. The power supplies were 'balanced' to minimize residuals. Figure 2 shows the difference orbits at a horizontal tune of 8.65. The tunes were changed from 8.65-8.75 to try to further reduce residuals. The minimal residuals are found over the 8.65-8.7 tune range, figure 3. At 8.75 they are approximately twice as large. Tests also showed that there is a $\sim 6 \mathrm{~mm}$ motion away from $\mathrm{H} 10$ due to these residuals, figure 4. If this proves to be a problem (the amplitude of the pulse required of the G10 kicker has to be increased to compensate) raising the H11A supply and lowering the G09A should reduce this motion at the expense of more residuals around the AGS

Measurements were done while holding the radius constant; the rf voltage was a bit low at $\sim 50 \mathrm{KV}$. G09A setpoint was at 1575, H09A at 1550 .

Changes in frequency, assuming constant beam momentum and guide magnet field, gives changes in circumference, which were looked for. As we are looking for a Hertz frequency change over a few ms, we actually look at the phase and note its rate of change relative to a reference oscillator. To check the 
change in phase, the rf was mixed with a $2226702 \mathrm{~Hz}$ oscillator. The difference frequency signals was filtered off and recorded. The mixed signal showed a maximum of $\sim 1^{\circ}$ with $0.5^{\circ}$ of noise over period of the $7 \mathrm{~ms}$ bump, figure 5. Thus the frequency is steady to less than a Hertz or the circumference to a fraction of a mm.

The rf phase error trace shows a maximum of $\sim 2.5^{\circ}$ twitch when the bumps were on and $\sim 1^{\circ}$ of noise when off, figure 6 . Thus the beam and $\mathrm{rf}$ are tracking and the beam frequency is to within a couple of Hertz and circumference to a $\mathrm{mm}$ or so. This phase bump is less than $5 \mathrm{mSec}$ long and $2.5^{\circ}$ implies a $1.5 \mathrm{KV} /$ turn acceleration giving a max energy change of $250 \mathrm{KV}$ or less than a part in $10^{\wedge} 4$. Time did not allow for observations at higher rf voltages.

Additionally, we wanted to check that turning these bumps on did not cause a significant change in the MMPS field. Gauss clock counts were recorded for $15 \mathrm{~ms}$ and $2000 \mathrm{~ms}$ windows the center of which is the time the bump is on, a steady change of $\sim-0.2 \mathrm{G}$ and $\sim 0.5 \mathrm{G}$ were seen respectively. The current error signal changed by $\sim 1 \mathrm{~A}$, corresponding to less than a 2 gauss bump, figure 7 . This is compared to a 15 gauss bump with the original configuration. From this we conclude the field $[\sim 8.5 \mathrm{Kg}]$ did not change by more than 2.5 parts in $10^{\wedge} 4$.

The above shift in momentum and field are probably the result of the radial loop keeping the radius fixed, thus mostly cancel; but as we do not know if the beam was accelerated to match the field jump, assume both errors add. The frequency shift would be a fraction of a hertz.

Thus the circumference may have changed half a millimeter with an error of a millimeter.

\section{Future Work}

Additional work is required to try to eliminate the horizontal excursions at $\mathrm{H} 10$ by adjusting the G09A and H11A supplies. Also of interest, is exploring the reproducibility of the vertical orbit displacement. These measurements should also be done for higher rf voltages.

L. Ahrens tells us that the measurements of phase shift might be improved and/or verified by using mountain range plots. He also suggests that the measurements might be done using the frequency meter at MCR3 with and without beam. His initial tests with the meter show that the analyzing power would be sufficient for our measurements. 

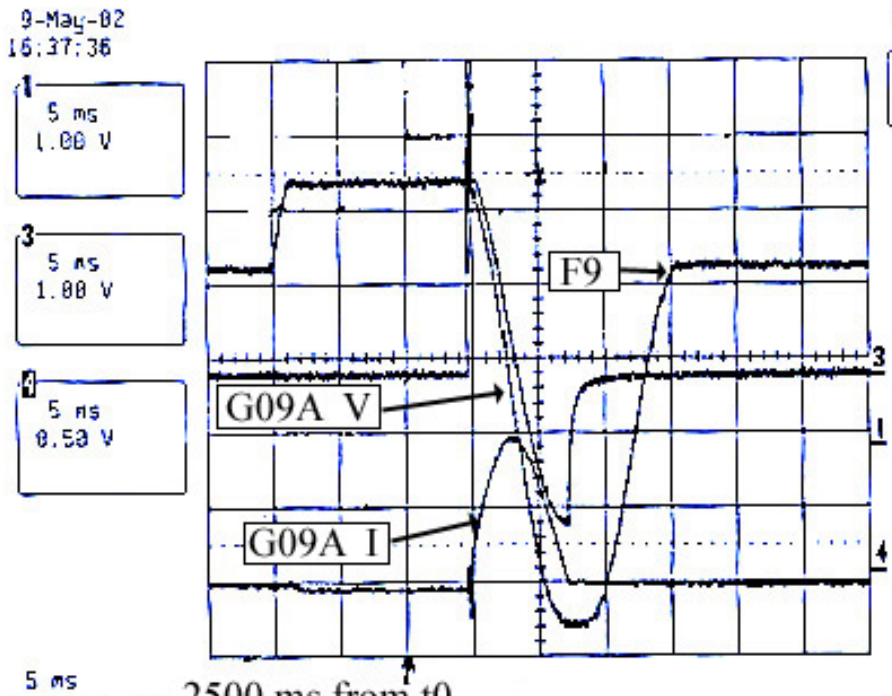

REEOTE EHABCE
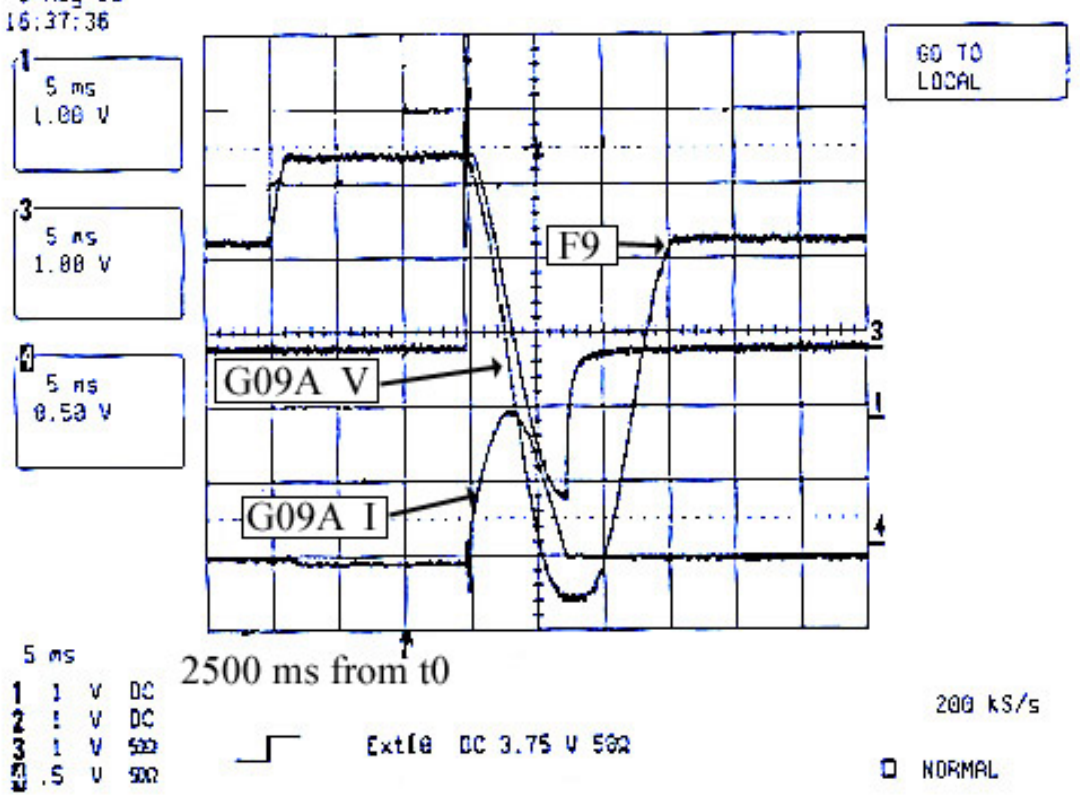

$200 \mathrm{kS} / \mathrm{s}$

(a)

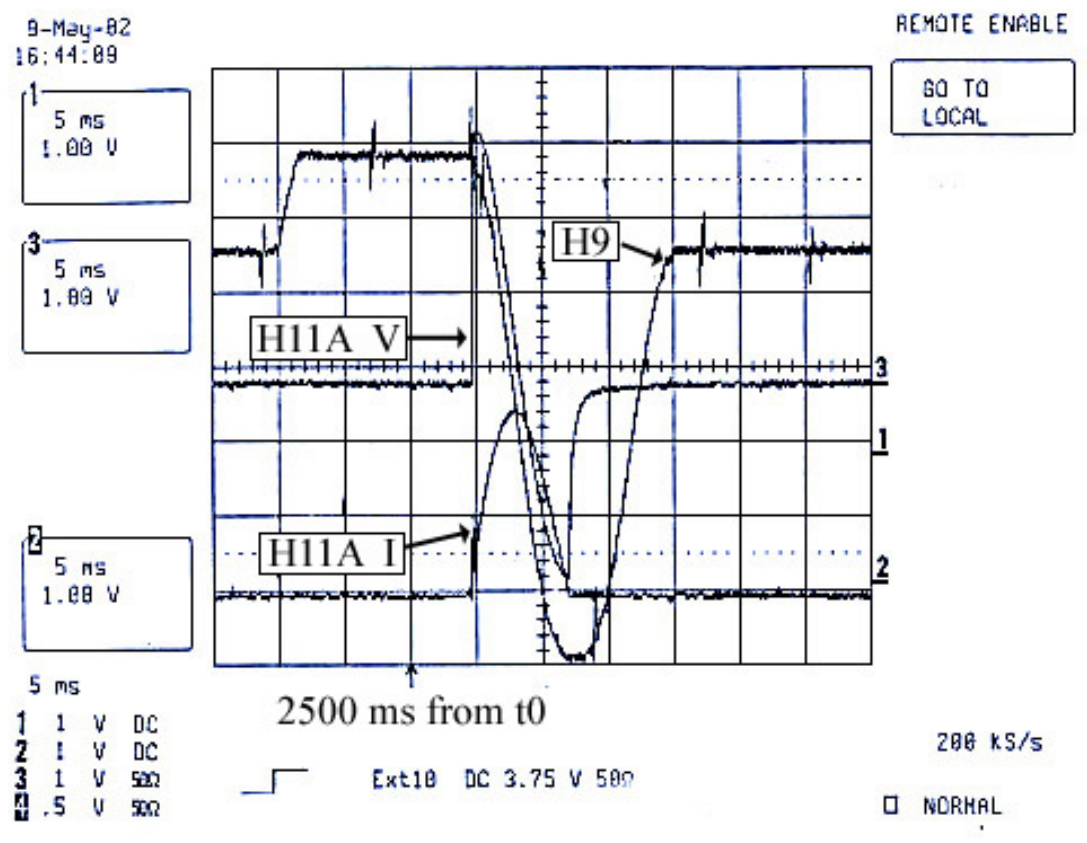

(b)

Figure 1. (a) Scope traces of G09A current, G09A voltage and BLWF09 (b) Scope traces of H09A current, H09A voltage and BLWH09. 


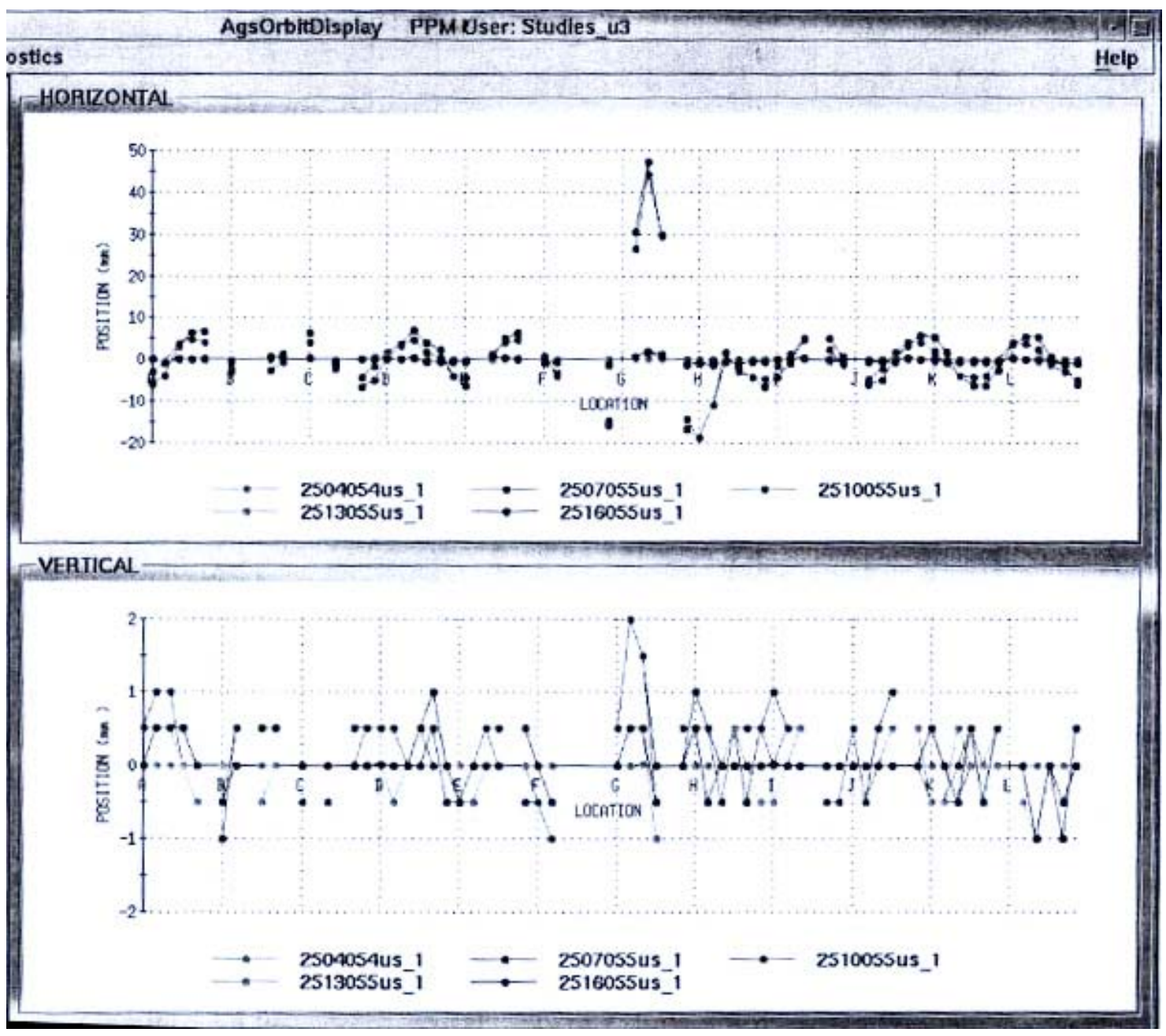

Figure 2. AGS orbit display with reference orbits.

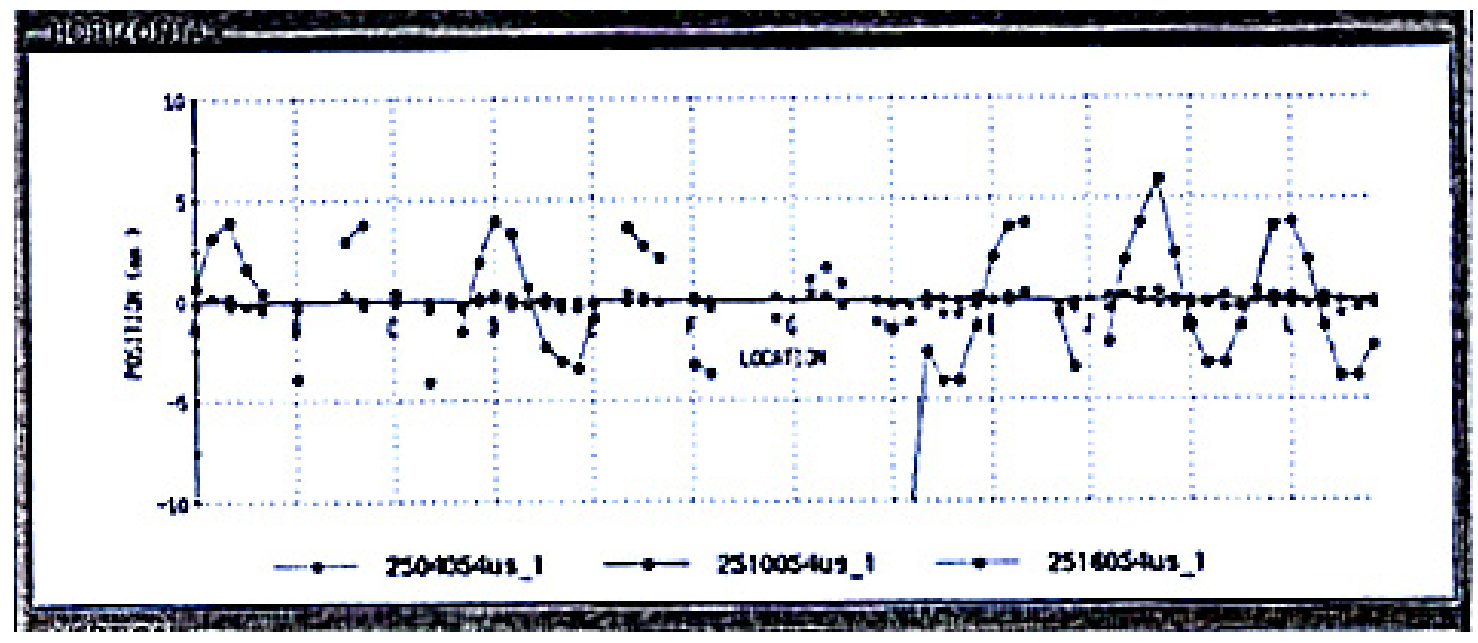

Figure 3. Closeup of orbit residuals at horizontal tune of 8.65. 


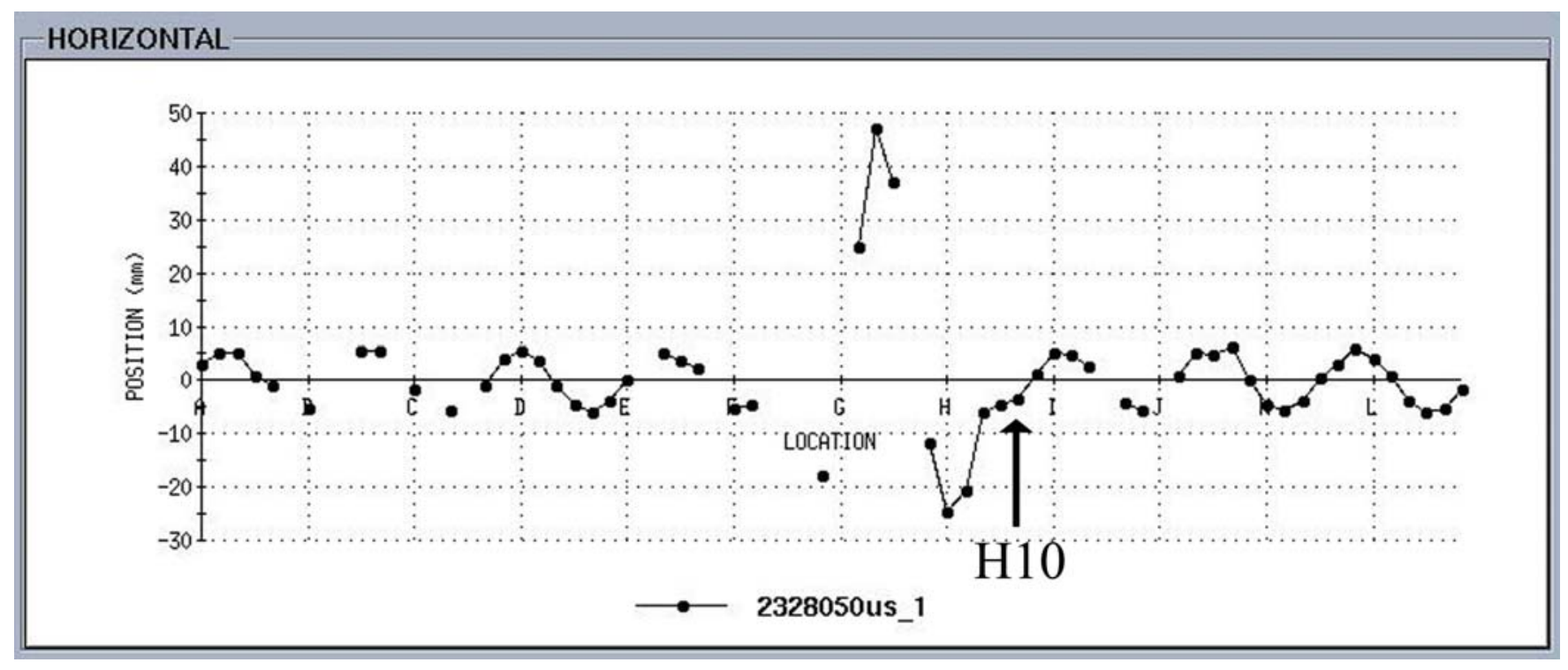

Figure 4. $\sim 6 \mathrm{~mm}$ motion away from $\mathrm{H} 10$ due to the residuals. 


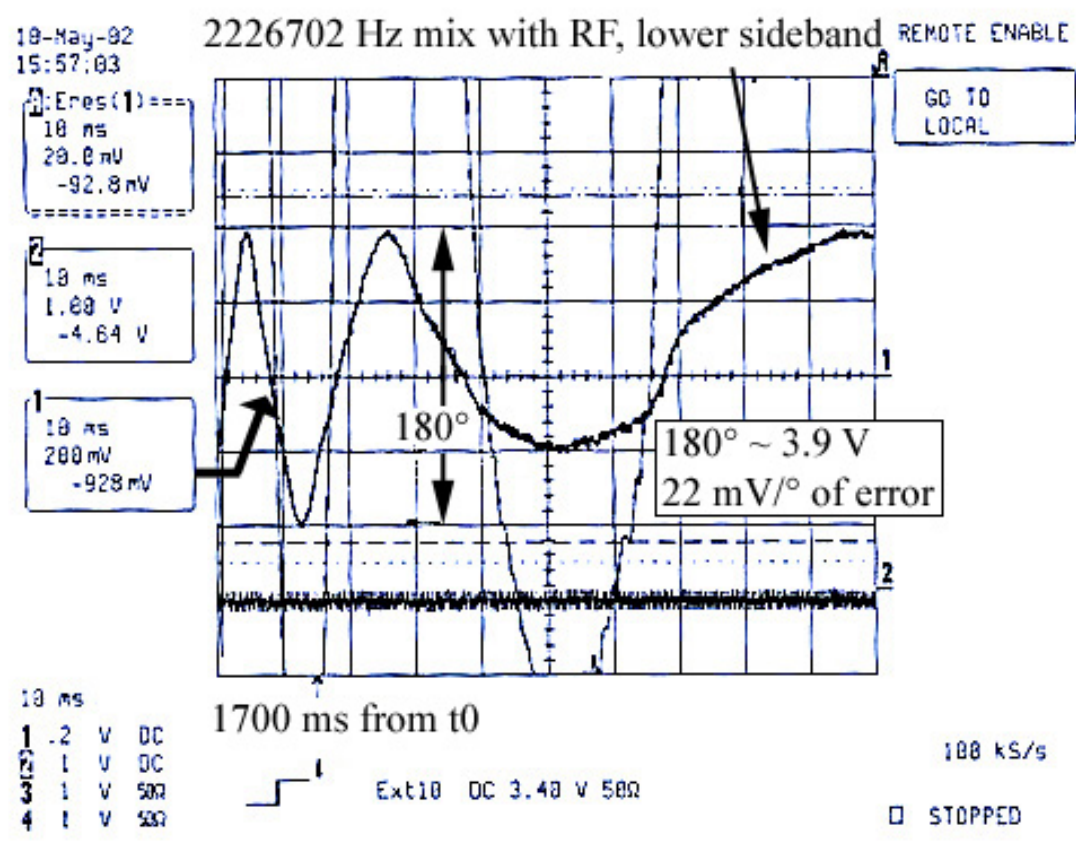

(a)

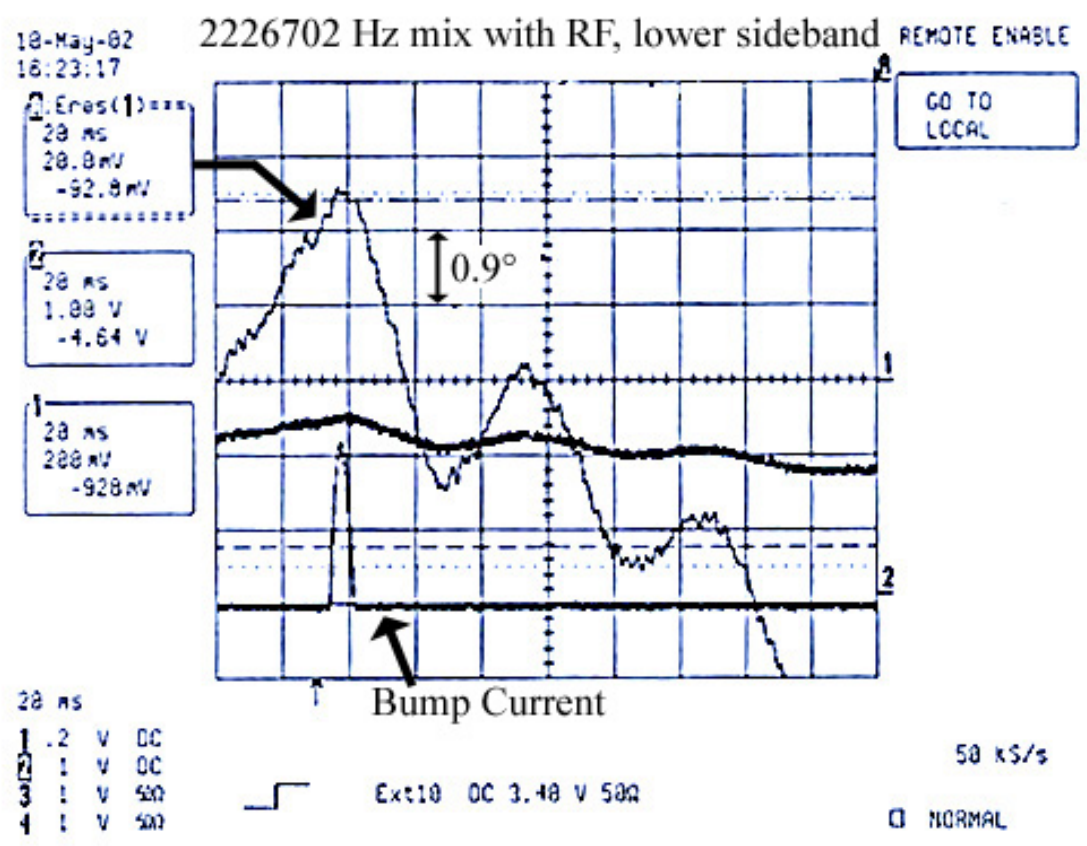

(b)

Figure 5. (a) Mix of $2226702 \mathrm{~Hz}$ oscillator frequency and rf phase error trace for calibration (b) Mix of $2226702 \mathrm{~Hz}$ oscillator frequency and rf phase error trace with beam showing a maximum of $1^{\circ}$ shift. 


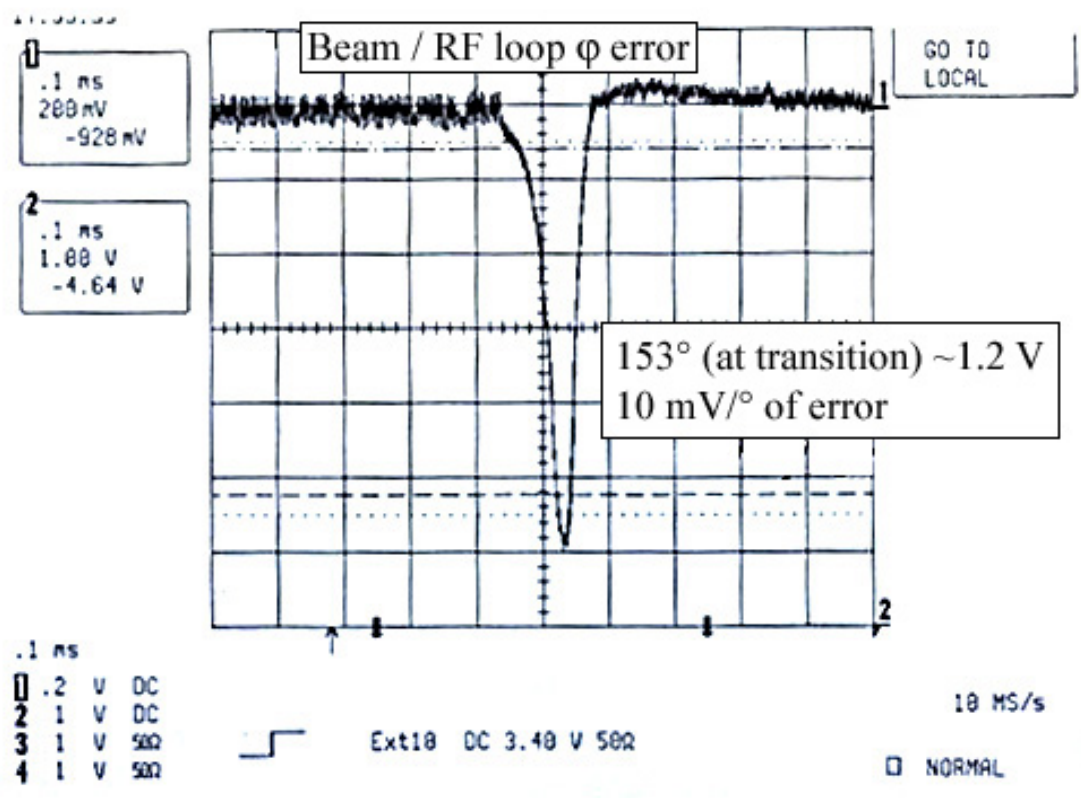

(a)

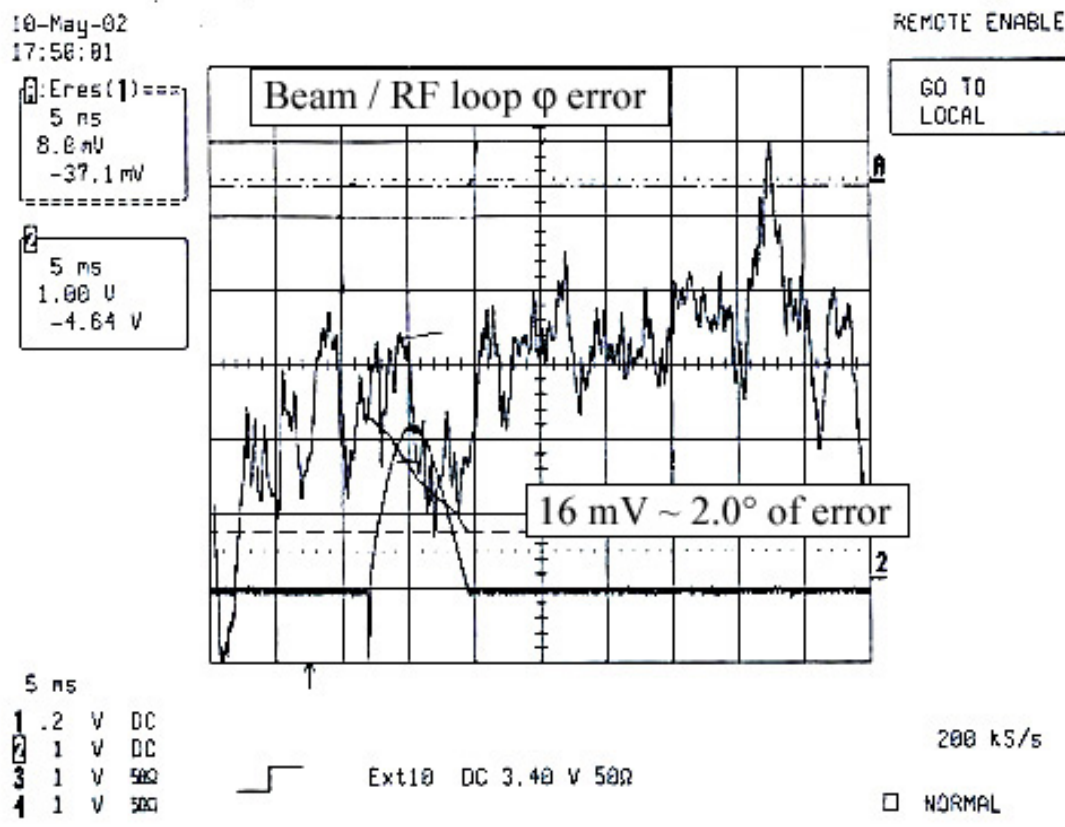

(b)

Figure 6. (a) RF phase error trace for calibration

(b) RF phase error trace with beam showing a maximum of $2.5^{\circ} \mathrm{shift}$. 

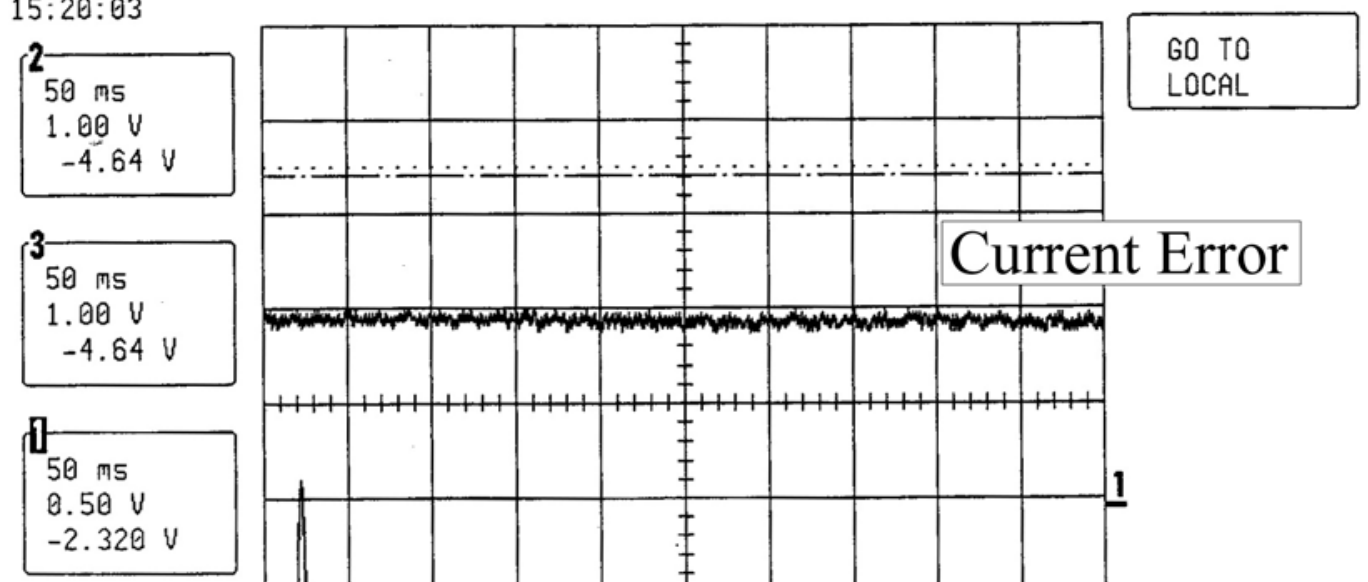

50 ms

1]. 5 V $\quad \mathrm{DC}$

Figure 7. Trace of the current error signal with the bump pulsing. 